\title{
Urothelium Patterns Bladder Smooth Muscle Location
}

\author{
MEI CAO, BENCHUN LIU, GERALD CUNHA, AND LAURENCE BASKIN
}

Department of Urology, University of California, San Francisco, San Francisco California 94143

\begin{abstract}
Smooth muscle differentiation is induced in the embryonic bladder by the centrally located urothelium in the undifferentiated mesenchyme in the periphery adjacent to the serosa. We hypothesize that under the appropriate signal the entire undifferentiated bladder mesenchyme is capable of smooth muscle differentiation and that the urothelium patterns fibromuscular development. Embryonic bladders of wild-type and Green Fluorescent Protein mice were separated into urothelial and mesenchymal components before smooth muscle differentiation (E12.5-E13). The urothelial layer green fluorescent protein was recombined and grafted with the mesenchyme (wild-type) in an orthotopic position, heterotopic position and ectopic position. In all cases, a zone of smooth muscle inhibition was observed adjacent to the epithelium whether the urothelium was in an orthotopic or heterotypic position. Bladder mesenchyme and bladder epithelium grafted alone did not grow. In conclusion, the full thickness of bladder mesenchyme is capable of smooth muscle differentiation dependent on the location of urothelium. These experiments support the hypothesis that urothelium secretes a diffusible factor that at high concentrations inhibits smooth muscle and at low concentrations induces smooth muscle, thus patterning mesenchymal cell differentiation across the full thickness of the fibromuscular bladder wall. (Pediatr Res 64: 352-357, 2008)
\end{abstract}

$\mathrm{T}$ The function of the urinary bladder is to store and expel urine at socially acceptable intervals while at all times maintaining a safe hydrostatic pressure to protect the kidneys. Mechanically, the bladder is composed of a urothelial barrier that limits urine permeability and allows mechanical expansion and contraction. The outer layer of the bladder is composed of smooth muscle necessary for efficient bladder emptying and low-pressure urine storage. Between the urothelial layer and smooth muscle layer is the lamina propria or submucosa, which is composed of loose fibrous connective tissue and allows the bladder to expand and collapse during filling and emptying.

Embryology. The bladder develops from the urogenital sinus (UGS), which is an endodermal tube, derived from the hindgut. The hindgut terminates as a caudal expansion called the cloaca. The uro-rectal septum subdivides the cloaca into the UGS ventrally and the rectum and anal canal dorsally. The UGS is further subdivided to give rise to the bladder cranioventrally at $12-13 \mathrm{~d}$ gestation in mice. From a technical prospective, the murine bladder can be identified macroscopically at gestational day 12

Received February 25, 2008; accepted April 29, 2008

Correspondence: Laurence Baskin, M.D., Department of Urology and Pediatrics, UCSF Children's Hospital, University of California, San Francisco, 400 Parnassus Ave. A640, San Francisco CA 94143; e-mail: lbaskin@urology.ucsf.edu

Supported by a grant from the NIH R01 DK073449. (term $\sim 20 \mathrm{~d}$ ). In humans, the process is similar but occurs at $\sim 7$ wk during the first trimester.

Epithelial-mesenchymal signaling. During development smooth muscle differentiates from bladder mesenchyme $(1,2)$. An epithelial signal is necessary for induction of bladder smooth muscle differentiation (Fig. 1) (1). Interestingly, the epithelial signal is not specific for age in that embryonic, newborn and adult urothelium all have the capacity to induce bladder smooth muscle differentiation (2). Furthermore, the bladder smooth-muscle-inducing signal is not specific to urothelium in that epithelial cells from organs such as uterus, gut and stomach, which have a thick smooth muscle wall, also can induce smooth muscle differentiation in bladder mesenchyme.

Epithelial-mesenchymal signaling is critical for organ and limb development. For example, the prostate, uterus, lung, kidney, intestine, limbs and the genital tubercle develop as a result of reciprocal mesenchymal-epithelial interactions in which epithelial signaling plays an important role (3-6).

Location and pattern of smooth muscle differentiation. Smooth muscle differentiation in the mouse urinary bladder begins at $13.5 \mathrm{~d}$ gestation (7). The hindgut and mid-gut develop smooth muscle at a similar stage (8). The uterus also forms smooth muscle (myometrium) from undifferentiated mesenchyme but much later at postnatal days 3-5 (9). In the gut and uterus, like the bladder, epithelial signaling is necessary for the induction of smooth muscle. Interestingly, smooth muscle in all three of these organs develops in concentric rings in the peripheral mesenchyme some distance away from the inducing epithelium. We have previously proposed that urothelium signals bladder mesenchyme via a diffusible factor along a concentration gradient (10). We propose that the factor from the urothelium is Sonic Hedgehog (Shh) (discussed later) (7). At high concentrations of Shh (close to the urothelium) bladder mesenchyme is inhibited from forming smooth muscle. At low Shh concentrations (away from the urothelium) smooth muscle is induced in the peripheral bladder mesenchyme. If this hypothesis is correct then we expect to be able to manipulate the signal that the mesenchyme receives from the urothelium by changing the location of the inducing urothelium (Fig. 2). For example, if the urothelium is placed on the serosal aspect of the bladder, 1) smooth muscle should differentiate some distance away from the ectopic urothelium in what would normally be the submucosa of the bladder, and 2) mesenchyme under the original serosal surface, which

Abbreviations: FVB, friend virus B-type; GFP, green fluorescent protein; K7, cytokeratin 7; Shh, sonic hedgehog; UGS, urogenital sinus 


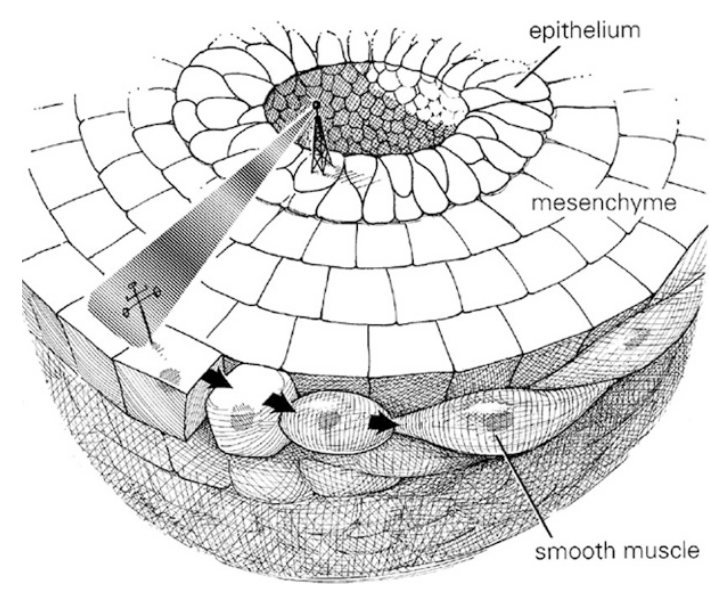

Figure 1. Cartoon of bladder epithelium inducing smooth muscle differentiation in the peripheral aspect of bladder mesenchyme, with a zone devoid of smooth muscle adjacent to the inducing urothelium.

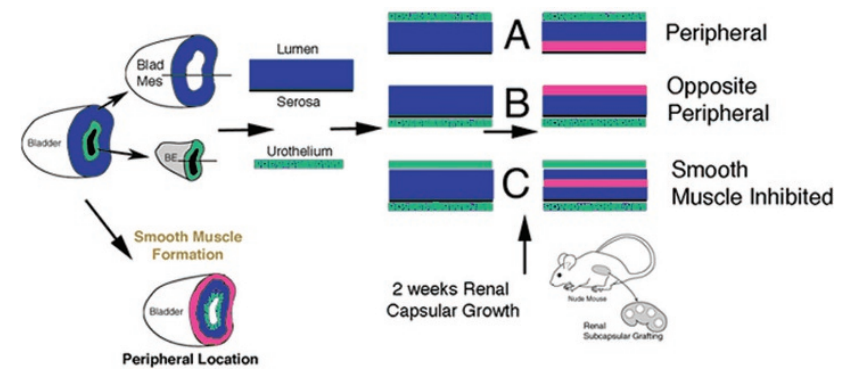

Figure 2. Schematic of bladder epithelial-mesenchymal tissue recombination experiments and expected results. Urothelium is represented in green, bladder mesenchyme in blue and bladder smooth muscle in pink.

normally differentiates into smooth muscle, should differentiate into loose fibrous connective tissue devoid of smooth muscle. Thus, the full thickness of undifferentiated bladder mesenchyme should be capable of forming or not forming smooth muscle depending on the location of the inducing epithelial signal.

\section{MATERIALS AND METHODS}

This study was approved by the UCSF institutional animal care and use committee. Timed pregnant friend virus B-type (FVB) mice were euthanized, and fetuses obtained on gestational day 12.5 or 13 (based on a plug date of zero). Smooth muscle develops in the mouse urinary bladder at E13.5. Timed-pregnant Green Fluorescent Protein (GFP) mice were euthanized, and fetuses obtained on gestational day 14 or 15 (based on a plug date of zero). Bladders were harvested by microdissection from each embryo and incubated in $0.02 \mathrm{M}$ ethylene-diamine-tetra-acetic acid for $20 \mathrm{~min}$ at $37^{\circ} \mathrm{C}$. The bladder epithelium (urothelium) was then separated from the bladder mesenchyme with forceps.

Tissue recombination experiments were performed by recombining epithelium with undifferentiated $13.5 \mathrm{~d}$ bladder mesenchyme using previously described techniques $(11,12)$. Six different experimental protocols were performed (see Fig. 2) (Table 1).

1. Normal orientation of the bladder epithelium (orthotopic fashion) in which GFP urothelium was placed back into the luminal area of the wild-type bladder mesenchyme before bladder smooth muscle differentiation.

2. Heterotypic orientation of the bladder epithelium in which GFP urothelium was placed on the serosal surface.

3. Orthotopic and heterotypic locations of the bladder epithelium in which GFP urothelium was recombined with undifferentiated bladder mesenchyme on both the prospective submucosal and prospective serosal surfaces.

4. Ectopic location of bladder epithelium using GFP urothelium implanted within E12.5-E13 bladder mesenchyme and/or intact bladders.

5. E12.5 or E13 bladder mesenchyme grafted alone (controls).

6. E14-E15 GFP bladder epithelium (urothelium) grafted alone (controls).

Additional experiments were performed with epithelia of nonurothelial origin. E13 wild-type bladder mesenchyme was recombined with: 1) postnatal day 3 GFP esophageal epithelium; 2) E14 GFP hindgut epithelium; 3) postnatal day 3 GFP uterine epithelium and 4) E14 GFP tail skin epithelium (Table 2).

The recombined and control specimens were grafted under the renal capsule of adult, male athymic mice (Harland Sprague Dawley, Indianapolis, IN). After 2 wk of growth, hosts were euthanized. The grafts were photographed and then dissected from the kidney. Grafts were fixed in formalin and embedded in paraffin. Sections of the grafts were stained with hematoxylin and eosin (H\&E). Immunohistochemical staining was performed as described below. The experiments were repeated multiple times (Table 1).

Immunohistochemical staining. Tissue sections were deparaffinized in histo-clear (National Diagnostic, Atlanta, GA) and hydrated in graded alcoholic solutions and distilled water. Antigen retrieval was carried out by microwave heating for $30 \mathrm{~min}$ in an antigen unmasking solution (Vector Laboratories, Foster City, CA). Endogenous peroxidase activity was blocked with $0.5 \%$ hydrogen peroxide in methanol for $30 \mathrm{~min}$ followed by washing in PBS pH 7.4. Normal goat serum was applied to the sections for $30 \mathrm{~min}$ to bind nonspecific sites. The sections were then incubated with the primary antibodies over-night at $4^{\circ} \mathrm{C}$ or with nonimmune mouse or rabbit IgG. Sections were then washed and incubated with biotinylated goat anti-mouse or goat antirabbit immunoglobulin (Sigma Chemical Co.) diluted with PBS at 1:200 for $30 \mathrm{~min}$ at room temperature. After incubation with the secondary antibody, sections were washed in PBS (three, 10 min washes) and then incubated with avidin-biotin complex (Vector laboratories, Foster City, CA) for $30 \mathrm{~min}$ at room temperature. Sections were then washed in PBS (three 10 min washes) before visualizing immunoreactivity using $3^{\prime}, 3^{\prime}$-diaminobenzidine in PBS and $0.03 \% \mathrm{H}_{2} \mathrm{O}_{2}$. Sections were counterstained with hematoxylin and dehydrated in alcohol.

The urothelial phenotype was characterized with an antibody to cytokeratin 7 (K7) and Uroplakin. A monospecific anti-cytokeratin antibody to K7 (mouse monoclonal) was a generous gift from Dr. E.B. Lane, University of Dundee, UK. Antibody to Uroplakin III was obtained from Nichirel Bioscience. GFP (rabbit polyclonal) was obtained from Santa Cruz Biotech. Antibody against smooth muscle alpha-actin is from Sigma Chemical Co. Biotinylated secondary antibodies were purchased from Amersham International (Arlington Heights, IL). For double staining with mouse and rabbit primary antibodies, fluorescein-conjugated anti-mouse and rhodamineconjugated anti-rabbit secondary antibodies (Dako, Carpinteria, CA) were used. To stain cell nuclei, sections were incubated with Hoechst dye 33258 (CalBiochem, La Jolla, CA).

\section{RESULTS}

Graft recovery for all tissue recombination experiments was greater than $90 \%$. The number of grafts recovered for each experiment is listed in Tables 1 and 2.

Table 1. Number of recovered grafts in each experiment composed of bladder mesenchyme \pm epithelia

\begin{tabular}{lccccc}
\hline \multicolumn{1}{c}{ Experiment } & 1 & 2 & 3 & 4 & 5 \\
E12.5-13 bladder mesenchyme & Orthotopic 10 & Heterotypic 9 & Orthotopic \& heterotypic 14 & Ectopic 9 & 4 \\
E12.5-13 intact bladder & & & 6 & 5 \\
Bladder mesenchyme alone & 3 & & & & 5 \\
Bladder epithelium alone & 3 & & & & \\
\hline
\end{tabular}


Table 2. Number of recovered grafts prepared with epithelia of nonbladder origin

\begin{tabular}{ccccc}
\hline & $\begin{array}{c}\text { Day 3 } \\
\text { GFP } \\
\text { esophagus }\end{array}$ & $\begin{array}{c}\text { E14 } \\
\text { GFP } \\
\text { hindgut }\end{array}$ & $\begin{array}{c}\text { Postnatal day 3 } \\
\text { GFP } \\
\text { uterus }\end{array}$ & $\begin{array}{c}\text { E14 } \\
\text { GFP } \\
\text { tail skin }\end{array}$ \\
\hline $\begin{array}{c}\text { E12.5-13 bladder } \\
\text { mesenchyme }\end{array}$ & 4 & 4 & 5 & 6 \\
\hline
\end{tabular}

1. When GFP urothelium was recombined with E12.5-E13 wild-type mesenchyme in the orthotopic location, smooth muscle developed in the normal location in the peripheral serosal aspect of the bladder wall (Fig. 3). In the submucosal zone, smooth muscle alpha-actin staining was not detected between the GFP urothelium and peripheral smooth muscle.

2. When GFP bladder urothelium was placed in the heterotypic position on the serosal surface of the bladder mesenchyme, smooth muscle developed in an abnormal position in the original prospective submucosa where smooth muscle normally does not form (Fig. 4). Correspondingly, smooth muscle development was inhibited immediately adjacent to the ectopic urothelium in the prospective serosal zone of bladder mesenchyme, which normally develops into smooth muscle.

3. When GFP bladder urothelium was placed on wild-type bladder mesenchyme in both the orthotopic and heterotypic locations, smooth muscle alpha-actin staining was expressed only centrally midway between the prospective submucosa and the prospective serosa (Figs. 5 and 6). Two zones of smooth muscle inhibition were seen as areas devoid of smooth muscle alpha-actin staining in close association with the orthotopic and heterotypic epithelia in the original prospective submucosal and prospective serosal zones.

4. When GFP urothelium was implanted in an ectopic location within an intact bladder or bladder mesenchyme alone, a zone of smooth muscle inhibition was also created in the peripheral bladder mesenchyme in close association with the ectopic epithelium in the area of the bladder that normally develops into smooth muscle (Fig. 7). Quadruple stain with GFP (green) urothelium, K7 (yellow), submucosa (Hoechst dye 33258) (blue) and smooth muscle alpha-actin (pink), respectively, clearly demonstrates a zone of smooth muscle inhibition by the ectopically implanted GFP urothelium.

$5 \& 6$. Isolated embryonic bladder mesenchyme (Fig. 8) or bladder epithelium (data not shown) grown in vivo for 2 wk did not exhibit growth, smooth muscle differentiation or epithelial proliferation consistent with past studies (2).

Epithelium isolated from the esophagus (Fig. 9A), hindgut (Fig. 9B), uterus (Fig. 9C), and tail skin (Fig. 9D) induced smooth muscle differentiation when recombined with E13 bladder mesenchyme. The amount of smooth muscle induced and zone of smooth muscle inhibition were different depending on the origin of the epithelium. Esophageal epithelium
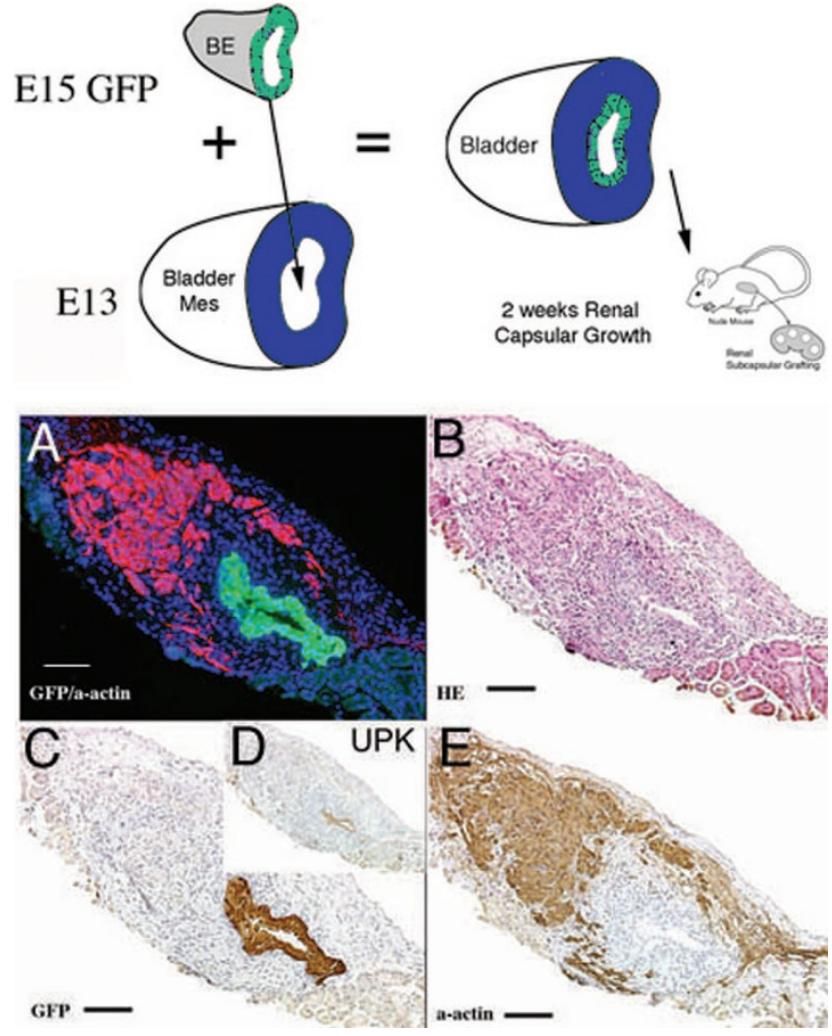

Figure 3. Schematic and results of urothelial recombination with bladder mesenchyme in the orthotopic location. Histologic serial sections: $(A)$ Color triple florescent stain, GFP is green, alpha-smooth muscle actin is pink and Hoescht dye is blue representing the zone of smooth muscle inhibition or submucosa; $(B) \mathrm{H} \& \mathrm{E}=$ hematoxylin and eosin; immunohistochemistry: $(C)$ $\mathrm{GFP}=$ green fluorescent protein; $(D) \mathrm{UPK}=$ uroplakin and $(E) \alpha$-actin $=$ smooth muscle alpha-actin. (magnification bar $=100 \mu \mathrm{m}$ ).

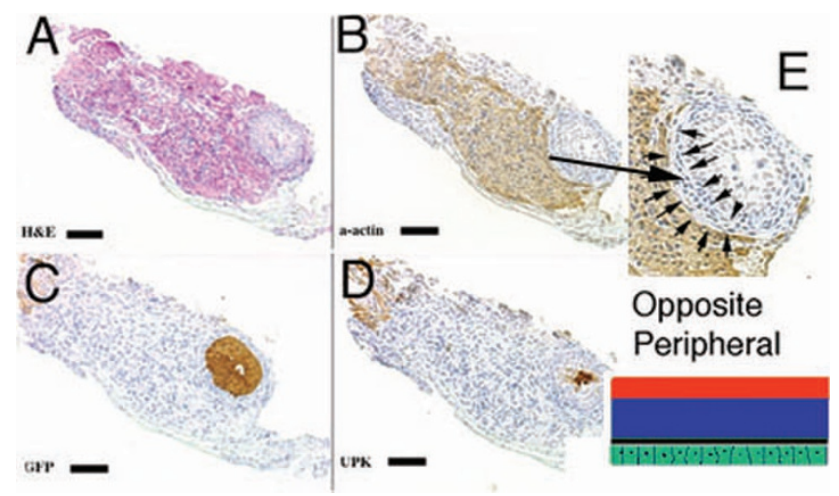

Figure 4. Schematic and results of urothelial recombination with bladder mesenchyme on the serosal surface of the bladder. Histologic serial sections: (A) $\mathrm{H} \& \mathrm{E}=$ hematoxylin and eosin; immunohistochemistry: $(B) \alpha$-actin $=$ smooth muscle alpha-actin; $(C)$ GFP $=$ green fluorescent protein; $(D)$ UPK = uroplakin; $(E)$ high power $(3 \times)$ of B. Note in $(E)$ the zone of smooth muscle inhibition (between the arrows) in the outer bladder mesenchyme, which normally differentiates into smooth muscle. (magnification bar $=100 \mu \mathrm{m}$ ).

induced less smooth muscle than that induced by epithelia of the hindgut, uterus and tail. Greater areas of smooth muscle inhibition (or larger amounts of submucosa) were observed when esophageal and hindgut epithelium was used in comparison to that seen when uterine or tail epithelia were used. 


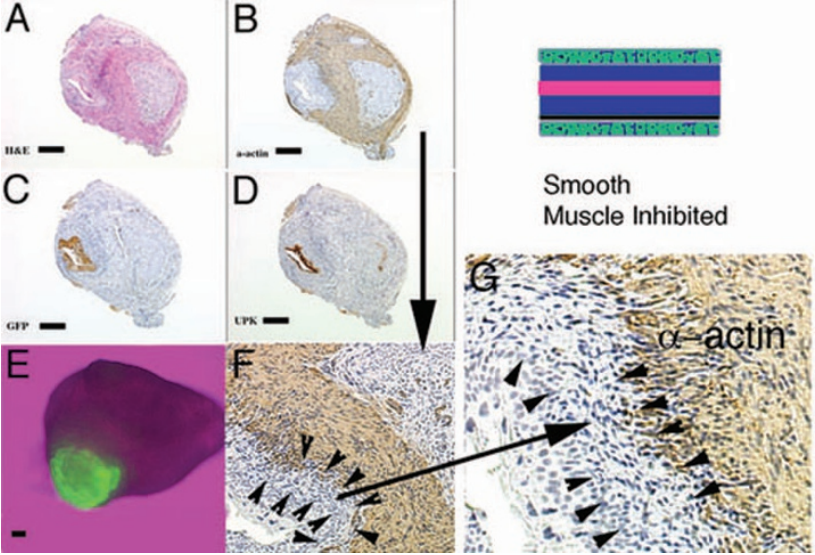

Figure 5. Schematic and results of urothelial recombination with an intact bladder in which ectopic urothelium was also placed in the serosal location, and thus both orthotopic and heterotypic urothelia were present. Histologic serial sections: (A) H\&E = hematoxylin and eosin; immunohistochemistry; (B) $\alpha$-actin $=$ smooth muscle alpha-actin; $(C)$ GFP $=$ green fluorescent protein; $(D)$ UPK = uroplakin; $(E)$ Gross image of the GFP-positive graft; $(F)$ higher magnification $(3 \times)$ of $(B, \alpha$-actin) showing two zones devoid of smooth muscle (between arrowheads and also large arrow); $(G)$ higher magnification $(2 \times)$ of $(F)$ showing urothelium, zone devoid of smooth muscle and $\alpha$-actin-positive smooth muscle. Note the zone of smooth muscle inhibition between the serosal ectopic urothelium and smooth muscle (between arrowheads). (magnification bar $=100 \mu \mathrm{m}$ ).

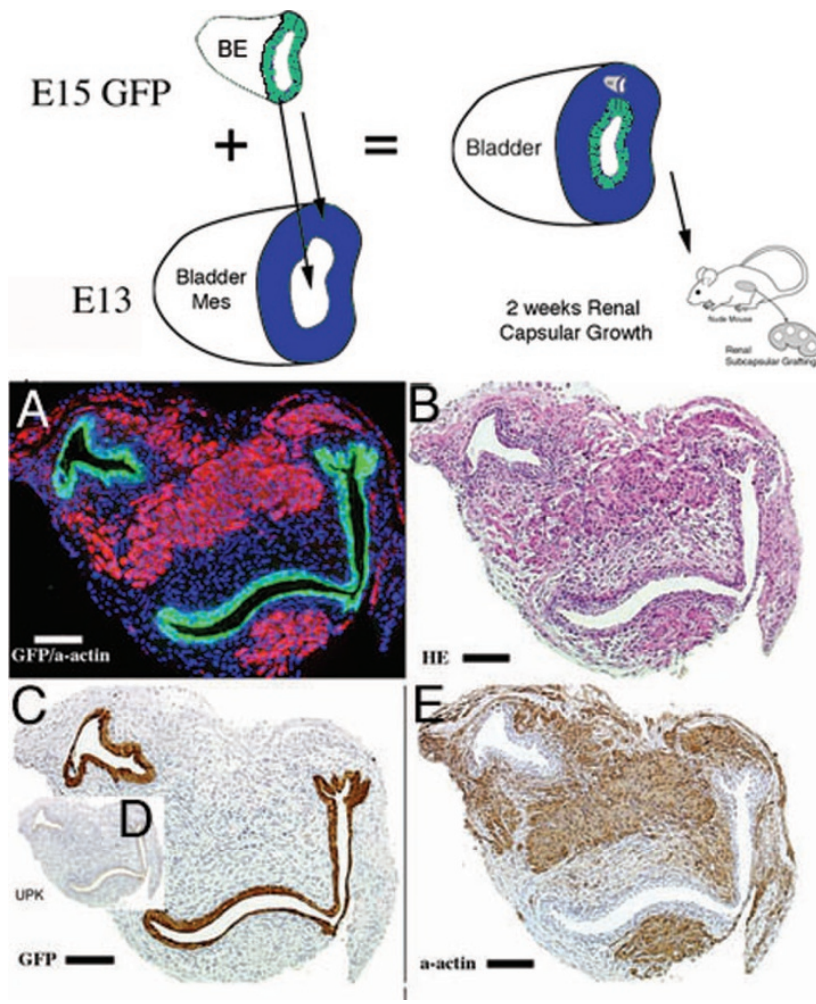

Figure 6. Schematic and results of urothelial recombination with bladder mesenchyme in heterotypic and orthotopic locations. Histologic serial sections: $(A)$ Color fluorescent triple stain, GFP is green, alpha-actin is pink and Hoescht dye is blue representing the zone of smooth muscle inhibition or submucosa around the placed urothelium; $(B) \mathrm{H} \& \mathrm{E}=$ hematoxylin and eosin; immunohistochemistry: $(C)$ GFP = green fluorescent protein; $(D)$ UPK = uroplakin and $(E) \alpha$-actin $=$ smooth muscle alpha-actin. (magnification bar $=$ $100 \mu \mathrm{m})$.
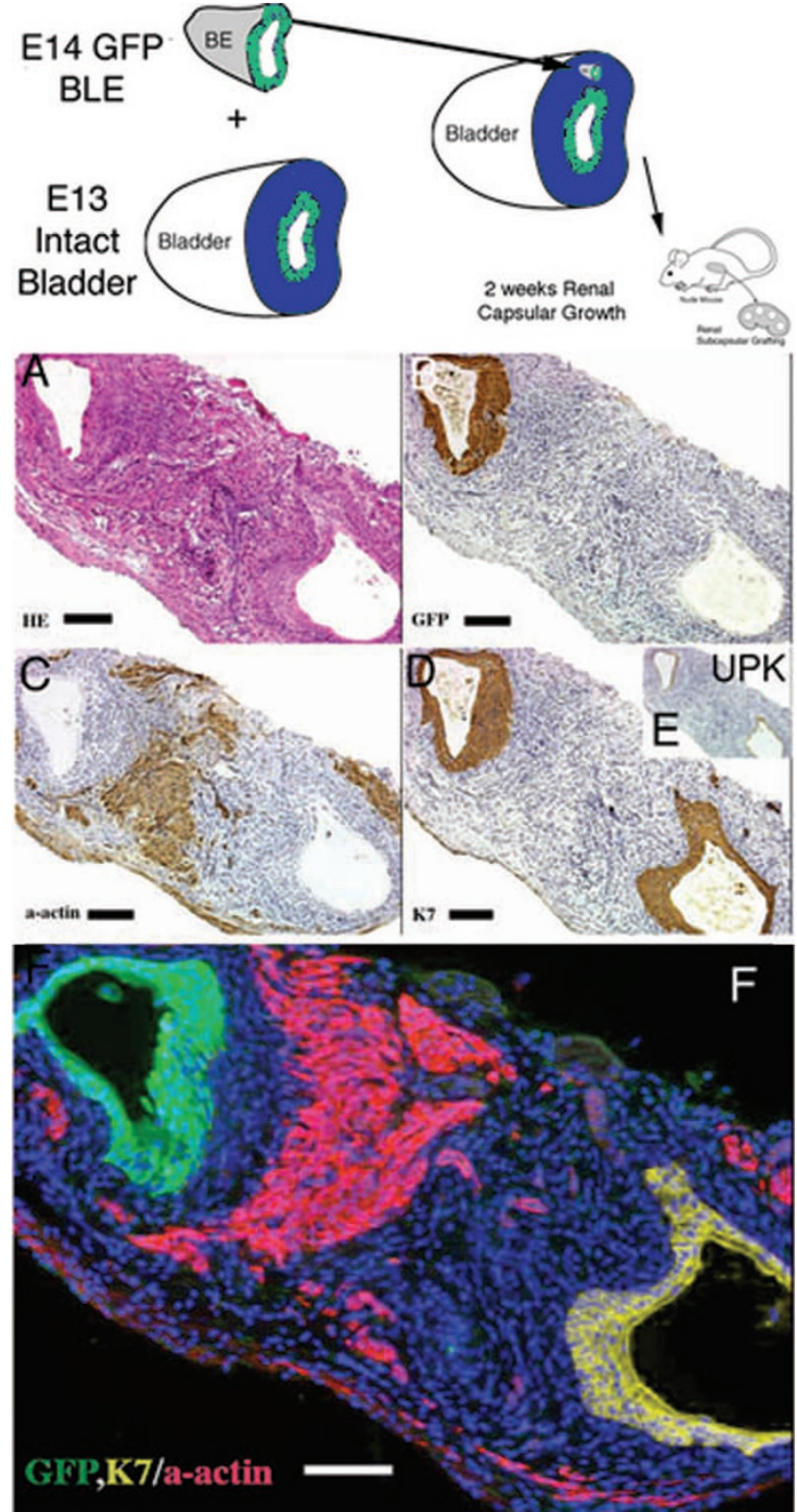

Figure 7. Schematic and results of ectopic urothelial recombination with an intact bladder. Histologic serial sections: $(A) \mathrm{H} \& \mathrm{E}=$ hematoxylin and eosin; immunohistochemistry; $(B)$ GFP = green fluorescent protein in brown; $(C)$ $\alpha$-actin $=$ smooth muscle alpha-actin in brown; $(D) \mathrm{K} 7=$ cytokeratin 7 in brown; UPK $=$ uroplakin in brown and $(F)$ color quadruple stain (note the zone of inhibition of smooth muscle represented by the blue Hoescht dye between the GFP urothelium in green, the alpha-actin smooth muscle in pink and native urothelium, $\mathrm{K} 7$ in yellow). (magnification bar $=100 \mu \mathrm{m}$ ).

\section{DISCUSSION}

Bladder smooth differentiation occurs in the peripheral (serosal) aspect of the bladder away from the inducing urothelium $(7,13)$. The hypothesis of our study is that the full thickness of bladder mesenchyme is capable of smooth muscle differentiation, the determining factor being the location of the inducing urothelial signal.

In this study, we have manipulated the location of the urothelial signal in respect to the bladder mesenchyme by placing the urothelium into its (a) normal orthotopic position in contact with the prospective submucosa, (b) placing it 


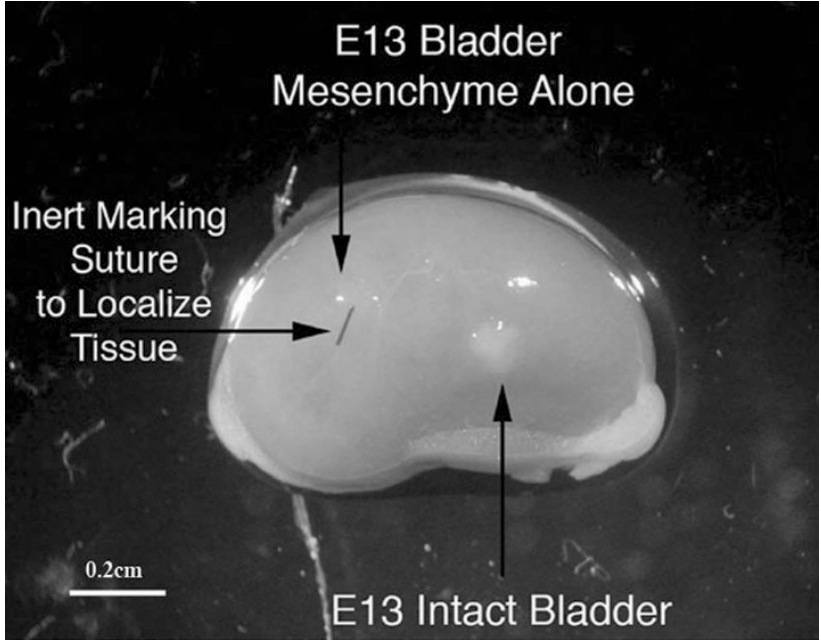

Figure 8. Bladder mesenchyme alone (control) grafted under the renal capsule does not grow (downward and horizontal arrow) as shown by the suture and lack of viable tissue compared with grafting of an E13 intact bladder (upward arrow) magnification bar $=0.2 \mathrm{~cm}$.

ectopically in contact with the prospective serosa, or (c) inserting it deep within the mesenchymal layer between the prospective submucosa and the prospective serosa (Fig. 2). We have shown that mesenchymal cells in all zones (prospective submucosa, prospective serosa, and in between can respond to urothelium by undergoing smooth muscle differentiation. For example, if the urothelial signal is replaced in the orthotopic submucosal position, smooth muscle forms in its normal location in the peripheral bladder mesenchyme (Fig. 3 ). Concomitantly, we observe the normal absence of smooth muscle in the submucosa zone close to the inducing urothelium where smooth muscle normally does not differentiate. We attribute this absence of smooth muscle to an inhibitory effect of urothelium.

Additional epithelial-mesenchymal experiments were designed to pattern the bladder mesenchyme based on various ectopic positions of the inducing epithelium. When the inducing urothelium is placed on the outer serosal surface of the bladder, the mesenchyme is induced to undergo smooth muscle differentiation, but the patterning is reversed (Fig. 4). In this case, a zone of smooth muscle inhibition was created in the prospective serosal zone next to the ectopic urothelium, and smooth muscle formed in the original submucosal zone of the bladder mesenchyme, which is normally devoid of smooth muscle (Fig. 4). If the inducing urothelium is placed on both sides of the bladder mesenchyme (luminal and serosal posi- tions), two submucosa areas or zones of inhibition are created, and the smooth muscle differentiates in the center of the bladder mesenchyme midway between the two urothelia (Fig. 5).

The effect of urothelial patterning is dramatically illustrated in the heterotypic/orthotopic experimental results shown in Figs. 6 and 7. The results show that urothelium induces smooth muscle a defined distance away from the inducing urothelium. Moreover, we consistently observe a zone of smooth muscle inhibition immediately adjacent to the inducing urothelium (Figs. 6 and 7).

Sonic hedgehog pathway. Previously, we have proposed that the inducing factor in the urothelium is Shh. This idea is supported by the observation that urothelium expresses Shh mRNA (7) and a Shh secreting cell line induces smooth muscle development in bladder mesenchyme (personal observation). Moreover, exogenous Shh induces smooth muscle differentiation in isolated bladder mesenchyme in vitro (personal observation). Shh can pattern tissue during development by forming a concentration gradient. Cells sense their position within the gradient and differentiate into distinct cell types as a function of Shh concentration as described in the developing neural tube and spinal cord (14-17). Cells are continuously exposed to complex regulatory signals and must interpret these signals to implement functional decisions. In many cases, cells are exposed to a variable or graded concentration of an external signal. At some intermediate threshold level of signal, cells switch between two alternate behaviors or fates. Important examples include cell survival versus apoptosis, cell proliferation versus differentiation and chemoattraction versus chemorepulsion.

The Shh network functions as a genetic switch with the ability to switch cell fate choices based on threshold concentrations of Shh (16). Thus, Shh is a prime candidate to act as both an inducer (lower concentration) as well as inhibitor (higher concentration) of bladder smooth muscle formation.

Sonic hedgehog signaling in the gut. During gut organogenesis the mesenchyme differentiates into distinct concentric layers around the endodermal epithelium forming the lamina propria/submucosa and outer smooth muscle layers. A thick smooth muscle layer and the enteric nerve plexus develop in the peripheral mesenchyme, always some distance from the epithelium, similar to bladder development. The concentric structures of the developing gut have been shown to be regulated by Shh derived from the endodermal gut epithelium
A
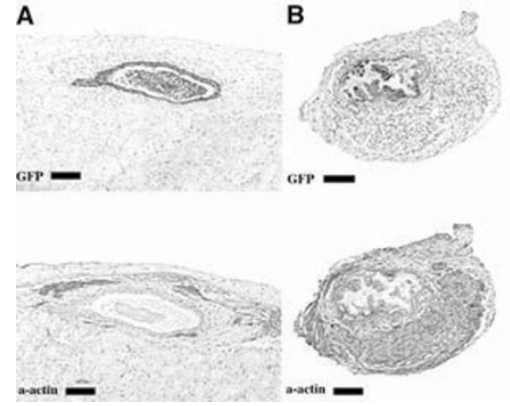

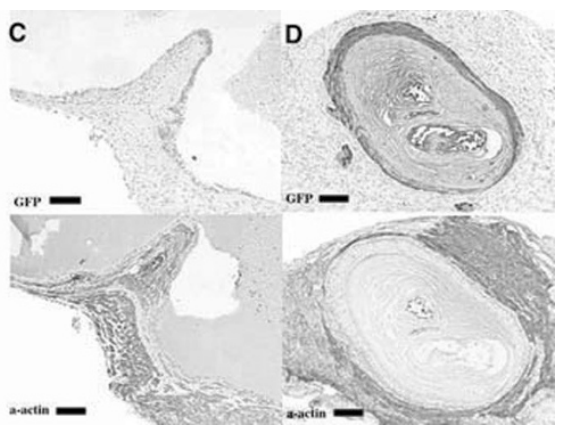

Figure 9. Schematic and results of epithelial recombination with bladder mesenchyme using epithelial cells from nonbladder origin. Immunohistochemistry: GFP = green fluorescent protein in brown (dark staining) (top row); $\alpha$-actin $=$ smooth muscle alpha-actin in brown (dark staining) (bottom row). (A) Esophageal, $(B)$ Hindgut, $(C)$ Uterus, $(D)$ Tail. (magnification bar $=100 \mu \mathrm{m}$ ) Note that the four epithelia of nonbladder origin all induced smooth muscle differentiation (discussed in text). 
(8). Specifically, it has been proposed that endodermal epithelium inhibits differentiation of smooth muscle and enteric neurons in adjacent submucosal mesenchyme. The endodermal epithelium activates expression of Patched and Bone morphogenetic protein 4 in adjacent nonsmooth muscle mesenchyme (lamina propria and submucosa) leading to inhibition of smooth muscle differentiation near the epithelium. Shh can mimic the effect of endodermal epithelium by inhibiting smooth muscle adjacent to the epithelium (high concentration) and inducing smooth muscle in the periphery in the developing gut (8).

Sonic hedgehog signaling in the ureter. The mesenchymal cells in the ureter, like the gut and bladder, express two fates dependent upon their location relative to the Shh expressing ureteral epithelium (18). Subepithelial ureteral mesenchymal cells, which are close to the Shh source, differentiate into fibroblastic cells, whereas the peripheral mesenchymal cells furthest away from the epithelium form smooth muscle. This is consistent with Shh acting as a paracrine signal, which promotes or inhibits mesenchymal cell differentiation into smooth muscle as a function of dose along a concentration gradient.

Sonic hedgehog in the bladder. Little is known about Shh expression and function in the bladder. Recent evidence has documented that Shh is expressed in bladder epithelium (urothelium) and ureteral epithelium (7,18-20). Moreover, Patched is expressed in the bladder mesenchyme. In patients with ano-rectal malformations it is thought that the $\mathrm{Shh} /$ Bone Morphogenetic protein 4 pathway is disturbed thus perturbing normal hindgut formation (21). Shh is clearly critical for bladder development since inactivation of Shh in the mouse has a severe embryonic lethal phenotype with cloacal exstrophy, penile agenesis and a deficiency of smooth muscle (22). Such a phenotype is consistent with the concept that Shh plays a major role in bladder smooth muscle development.

Epithelial patterning from nonurothelial cell origin. Before the differentiation of smooth muscle in the embryonic bladder, isolated bladder mesenchyme fails to undergo smooth muscle differentiation even after prolonged periods of growth (Fig. 8). Herein, we have shown that a urothelial signal is necessary to both induce smooth muscle as well as pattern smooth muscle differentiation within the bladder mesenchyme. The inducing/inhibitory signal is not specific to urothelium since epithelia of nonurothelial origin can also induce bladder smooth muscle (2). In this regard, we tested the ability of four nonurothelial epithelia to induce and pattern smooth muscle differentiation in bladder mesenchyme. As noted above, the mesenchyme of the gut and uterus also form smooth muscle in concentric rings in the peripheral aspect of the organ. Figure 9 illustrates that the amount of smooth muscle and zone of smooth muscle inhibition were different depending on the origin of the epithelium. Esophageal epithelium tended to induce less smooth muscle than hindgut, uterus and tail. Esophageal and hindgut epithelium tended to have greater areas of smooth muscle inhibition or larger submucosa areas than that observed with uterine or tail epithelia. Presumably, the hedgehog family of genes is the inducing agent resulting in both induction and patterning of smooth muscle in these other organs. In conclusion, the full thickness of bladder mesenchyme is capable of smooth muscle differentiation dependent on the location of urothelium. Based upon previous studies, we propose that urothelium secretes a diffusible factor, Shh, which at high concentrations inhibits smooth muscle and at low concentrations induces smooth muscle, thus patterning mesenchymal cell differentiation across the full thickness of the fibromuscular bladder wall.

\section{REFERENCES}

1. Baskin LS, Hayward SW, Young P, Cunha GR 1996 Role of mesenchymalepithelial interactions in normal bladder development. J Urol 156:1820-1827

2. DiSandro MJ, Li Y, Baskin LS, Hayward S, Cunha G 1998 Mesenchymal-epithelia interactions in bladder smooth muscle development: epithelial specificity. J Urol 160:1040-1046

3. Haffen K, Kedinger M, Simon-Assmann P 1987 Mesenchyme-dependent differentiation of epithelial progenitor cells in the gut. J Pediatr Gastroenterol Nutr 6:14-23

4. Cunha GR, Young P, Brody JR 1989 Role of uterine epithelium in the development of myometrial smooth muscle cells. Biol Reprod 40:861-871

5. Cunha GR, Battle E, Young P, Brody JR, Donjacour A, Hayashi N 1992 Role of epithelial-mesenchymal interactions in the differentiation and spatial organization of visceral smooth muscle. Epithelial Cell Biol 1:105-118

6. Kurzrock EA, Baskin LS, Li Y, Cunha GR 1999 Epithelial-mesenchymal interactions in development of the mouse fetal genital tubercle. Cells Tissues Organs 164:125-130

7. Shiroyanagi Y, Liu B, Cao M, Agras K, Li J, Hsieh MH, Baskin LS 2007 Urothelial sonic hedgehog signaling plays an important role in bladder smooth muscle formation. Differentiation 75:968-977

8. Sukegawa A, Narita T, Kameda T, Saitoh K, Nohno T, Iba H 2000 The concentric structure of the developing gut is regulated by sonic hedgehog derived from endodermal epithelium. Development 127:1971-1980

9. Brody JR, Cunha GR 1989 Histologic, morphometric, and immunocytochemica analysis of myometrial development in rats and mice: I. Normal development. Am J Anat 186:1-20

10. Liu W, Li Y, Cunha S, Hayward G, Baskin LS 2000 Diffusable growth factors induce bladder smooth muscle differentiation. In Vitro Cell Dev Biol Anim 36:476484

11. Li Y, Liu W, Hayward SW, Cunha GR, Baskin LS 2000 Plasticity of the urothelial phenotype: effects of gastro-intestinal mesenchyme/stroma and implications for urinary tract reconstruction. Differentiation 66:126-135

12. Cunha GR, Battle E, Young P, Brody J, Donjacour A, Hayashi N 1992 Role of epithelial-mesenchymal interactions in the differentiation and spatial organization of visceral smooth muscle. Epithelial Cell Biol 1:76-83

13. Baskin LS, Hayward SW, Sutherland RA, DiSandro MJ, Thomson AA, Goodman J 1996 Mesenchymal-epithelial interactions in the bladder. World J Urol 14:301-309

14. Ericson J, Muhr J, Jessell TM, Edlund T 1995 Sonic hedgehog: a common signal for ventral patterning along the rostrocaudal axis of the neural tube. Int J Dev Biol 39:809-816

15. Hynes M, Porter JA, Chiang C, Chang D, Tessier-Lavigne M, Beachy PA 1995 Induction of midbrain dopaminergic neurons by sonic hedgehog. Neuron 15:35-44

16. Lai K, Kaspar BK, Gage FH, Schaffer DV 2003 Sonic hedgehog regulates adult neural progenitor proliferation in vitro and in vivo. Nat Neurosci 6:21-27

17. Ericson J, Muhr J, Placzek M, Lints T, Jessell TM, Edlund T 1995 Sonic hedgehog induces the differentiation of ventral forebrain neurons: a common signal for ventral patterning within the neural tube. Cell 81:747-756

18. Yu J, Carroll TJ, McMahon AP 2002 Sonic hedgehog regulates proliferation and differentiation of mesenchymal cells in the mouse metanephric kidney. Development 129:5301-5312

19. Bitgood MJ, McMahon AP 1995 Hedgehog and Bmp genes are coexpressed at many diverse sites of cell- cell interaction in the mouse embryo. Dev Biol $172: 126-138$

20. Karavanova ID, Dove LF, Resau JH, Perantoni AO 1996 Conditioned medium from a rat ureteric bud cell line in combination with bFGF induces complete differentiation of isolated metanephric mesenchyme. Development 122:4159-4167

21. Sasaki Y, Iwai N, Tsuda T, Kimura O 2004 Sonic hedgehog and bone morphogenetic protein 4 expressions in the hindgut region of murine embryos with anorectal malformations. J Pediatr Surg 39:170-173

22. Yucel S, Liu W, Cordero D, Donjacour A, Cunha GR, Baskin LS 2004 Anatomical studies of the fibroblast growth factor-10 mutant, Sonic Hedge Hog mutant and androgen receptor mutant mouse genital tubercle. Adv Exp Med Biol 545:123-148 